\title{
Trends in Cancer
}

\section{Review}

\section{The Unfolded Protein}

\section{Response in Immunogenic Cell Death and Cancer Immunotherapy}

\author{
Nicole Rufo, ${ }^{1}$ Abhishek D. Garg, ${ }^{1}$ and Patrizia Agostinis ${ }^{1, \star}$
}

The unfolded protein response (UPR) is a conserved pathway that is stimulated when endoplasmic reticulum (ER) proteostasis is disturbed or lost. Accumulating evidence indicates that chronic activation of the UPR supports the main hallmarks of cancer by favoring cancer cell-autonomous and nonautonomous processes, which ultimately foster the immunosuppressive and protumorigenic microenvironment. However, certain forms of therapy-induced ER stress can elicit immunogenic cancer cell death (ICD), which enables the release of key immunostimulatory or danger signals, eventually driving efficient antitumor immunity. In this review, after a brief discussion of the interplay between ER stress and protumorigenic inflammation, we review the relevance of therapymediated ER stress pathways in evoking ICD and how they could be used to optimize current immunotherapy approaches against cancer.

\section{Endoplasmic Reticulum Stress-Induced Unfolded Protein Response}

The endoplasmic reticulum (ER) is a crucial organelle that extends throughout the volume of the cytoplasm, mainly serving a threefold purpose: (i) calcium $\left(\mathrm{Ca}^{2+}\right)$ storage and buffering, (ii) lipid biosynthesis, and (iii) productive folding and assembly of secretory and transmembrane proteins (assisted by the agency of a variety of molecular chaperones). Indeed, the ER represents the first compartment of the secretory pathway and about one-third of the polypeptides synthesized by a cell enter the ER lumen, where they undergo different maturation steps (e.g., folding, glycosylation, and disulfide bond formation), thus ensuring that only properly folded proteins exit the ER and reach their final destination [1]. If ER quality control mechanisms fail, misfolded proteins exit the ER and are degraded through the ER-associated degradation pathway or autophagy. Since a single organelle orchestrates these processes pivotal for cellular survival and homeostasis, it is not surprising that ER-associated perturbations have critical consequences for the overall biochemistry and fate of intracellular and extracellular milieus of a cell. Such perturbations include (i) ER protein overloading either due to increased protein translation or inhibition of degradation of misfolded proteins; (ii) hypoxia and/or glucose/nutrient deprivation; (iii) disruption of the ER-Ca ${ }^{2+}$ pool or buffering capacity; and (iv) altered redox regulation of the highly oxidative ER lumen, which impairs the formation of disulfide bonds [2]. Deregulation of ER homeostasis (caused by both intracellular and extracellular stressors) underlies several diseases, including (but not limited to) metabolic and inflammatory diseases, neurodegeneration, retinal dystrophies, cystic fibrosis, and cancer [3].

Considering the relevance of maintaining ER homeostasis, cells have developed a major, evolutionarily conserved adaptation mechanism to rapidly sense and adequately respond

\section{Trends}

The ER is a key organelle in cell physiology, and it evolved as an elaborated signaling pathway to cope with life-threatening perturbations of its homeostatic state. This process, called the UPR, is exploited by cancer cells to survive in their microenvironment and to promote tumor progression.

Many approaches have been investigated to inhibit or exacerbate UPR to kill cancer cells. Among these, a defined set of agents or procedures can induce a form of ER stressmediated cancer cell death that is immunogenic (the so-called ICD), generating an effective antitumor immunity.

ICD is characterized by a spatiotemporally defined emission of DAMPs that establish a productive interface with immune cells.

Combinatorial strategies based on ICD and immune checkpoint blockade are currently being investigated.

${ }^{1}$ Cell Death Research \& Therapy (CDRT) Lab, Department of Cellular and Molecular Medicine, KU Leuven (University of Leuven), Leuven, Belgium 


\section{Trends in Cancer}

to perturbations in the ER folding machinery, called the unfolded protein response (UPR). The UPR aims to restore the ER-associated protein folding capacity by increasing ER volume and expression of ER-associated chaperones, as well as (transiently) attenuating global protein translation. In mammalian cells, the UPR is governed by three ER stress sensors, namely, inositol-requiring enzyme 1 alpha (IRE1 $\alpha$ ), protein kinase R-like ER kinase (PERK), and activating transcription factor 6 (ATF6) [4]. In physiological conditions, these proteins are kept in an inactive state by the master regulator of the UPR, that is, the glucose-regulated protein (GRP)-78 (also known as BiP) [5]. However, in case of loss of ER proteostasis or $\mathrm{Ca}^{2+}$ dysregulation, BiP disassociates from these ER stress sensors, thereby activating the UPR signaling pathway (Figure 1). Overall, when the ER stress is of mild intensity, the UPR facilitates cell-autonomous re-establishment of cellular homeostasis and survival, as well as inflammatory responses aimed at tissue turnover or repair. However, when ER stress intensity is too severe (thereby rendering a repair and rescue program obsolete), the UPR engages signaling pathways culminating into cell death, which are driven by the coordinated (transcriptionally regulated) actions of ATF4 and C/EBP homologous protein (CHOP), downstream the PERK pathway (Figure 1) $[6,7]$. In specific contexts, the activation of inflammatory transcriptional programs and/or danger signaling pathways may accompany lethal UPR signaling, aiming to alert the immune system about diseased or damaged cells (discussed later). Although cell death mediated by ER stress is mainly executed through the apoptotic program [2], recent studies have linked loss of ER homeostasis also to various forms of regulated necrosis, such as necroptosis [8] and ferroptosis [9]. An in-depth discussion of the molecular mechanisms underlying these emerging regulated cell death modalities and their intersection with the immune system is beyond the scope of this review. In addition, how these cell death pathways modulate ER stress-mediated extrinsic responses, such as inflammation and immunity, has not been elucidated yet.

This coordinated regulation of cell fate and communication with extracellular entities is of great importance for cancer progression and anticancer therapy response, especially immunotherapy. In this review, we briefly examine the ER stress-associated signaling pathways regulating tumor-associated inflammation and antitumor immunity. We primarily focus on the relevance of therapeutic modulation of ER stress [especially in the context of immunogenic cell death (ICD)] in the cancer cells and how this may influence the overall susceptibility or resistance to cancer immunotherapy. The role of UPR in immune cells regulating crucial immunomodulatory and inflammatory responses within the tumor microenvironment (TME) will not be discussed here but has been addressed in excellent recent reviews $[10,11]$.

\section{ER Stress-Driven Protumorigenic Inflammation}

Tumor cells, to grow and proliferate, need to survive in a deeply hostile (micro)environment. Several recent reviews highlight that this peculiar condition evokes chronic ER stress, and that UPR is exploited by cancer cells not only for survival, but also to promote tumor growth and progression by supporting nearly all the (established) hallmarks of cancer [12-15]. Adaptation to the increasingly stressed TME requires a plastic process enabling cancer cells to coevolve with the tumor stroma while maintaining an enhanced ability to survive and outgrow. Overactivation of the UPR provides a clear survival advantage to cancer cells since beyond supporting their oncogene-driven, increased synthetic and secretory demand, it fosters crucial remodeling of the TME and proinflammatory pathways with profound autocrine and paracrine impact on stromal and immune cells $[4,11,16,17]$. Indeed, several pieces of evidence have disclosed the ability of cancer cell-associated UPR [in particular, the PERK-ATF4 and IRE1 $\alpha-X$ box binding protein 1 (XBP1) pathways] to release vascular endothelial growth factor and other proangiogenic cytokines, thus interfacing with the endothelial cells, leading to dysfunctional and tumor-supporting angiogenesis $[13,18]$. 


\section{Trends in Cancer}

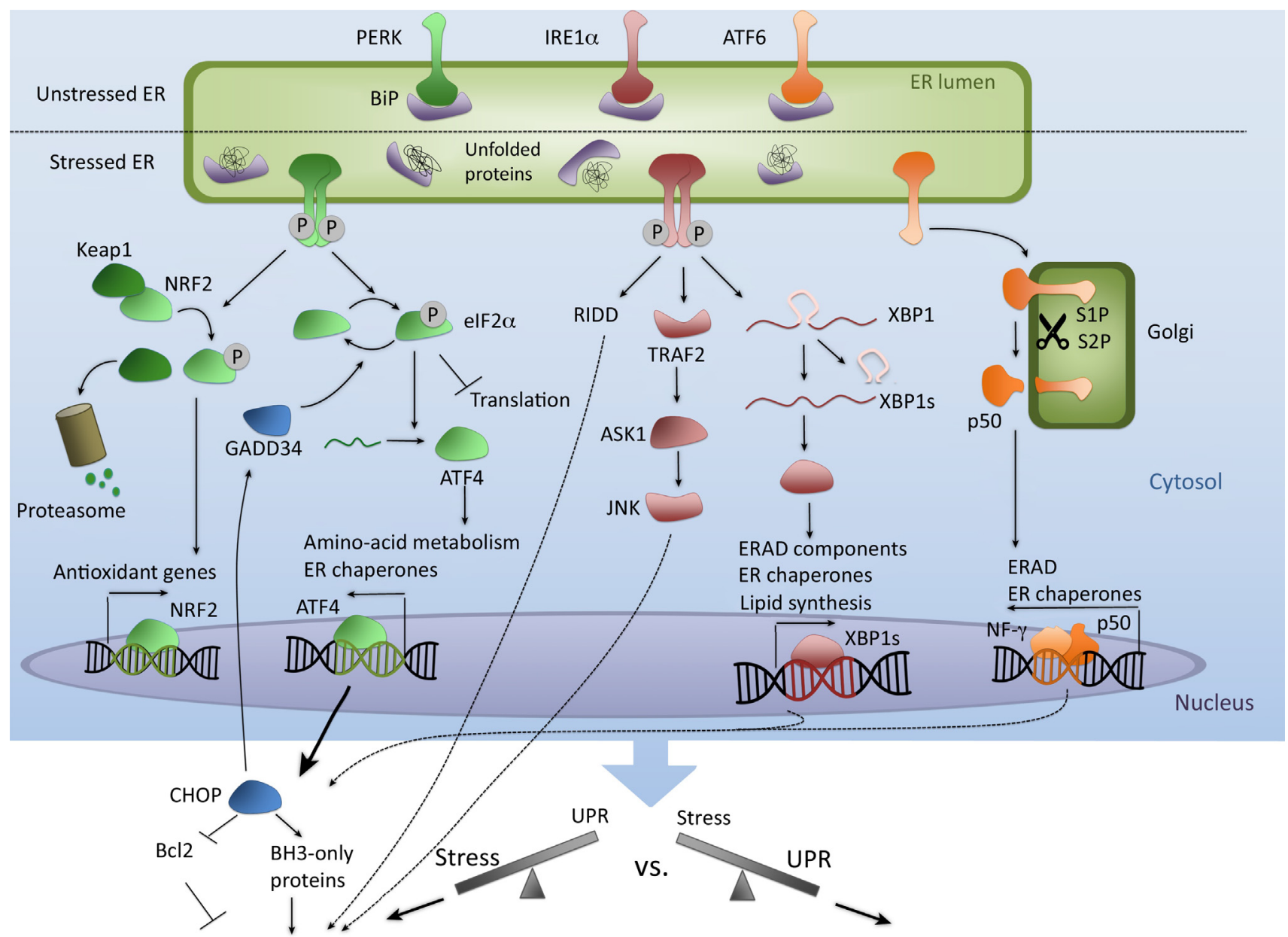

Apoptosis

Survival

Trends in Cancer

Figure 1. Signaling through the Unfolded Protein Response (UPR). When intra/extracellular stressors lead to misfolded proteins in the ER, BiP binds to these unleashing the three UPR sensors (PERK, IRE1 $\alpha$, and ATF6, reviewed in [83]). PERK dimerizes, autophosphorylates, and in turn phosphorylates elF2 $\alpha$ that inhibits protein synthesis to alleviate ER protein synthesis burden, with the exception of specific mRNAs, such as ATF4. PERK may also trigger the dissociation of Kelch-like ECH-associated protein 1 (Keap1) from nuclear factor erythroid 2-related factor (NRF2). IRE1 $\alpha$ dimerizes and transautophosphorylates, activating its RNase domain. Active IRE1 $\alpha$ splices XBP1 mRNA to form the more stable XBP1s mRNA, which encodes a potent transcription factor. IRE1 $\alpha$ can also degrade a subset of mRNAs through a process known as regulated IRE1 $\alpha$-dependent decay (RIDD) of mRNA. ATF6 translocates to the Golgi where it is cleaved, and then its p50 cytoplasmic fragment heterodimerizes with the nuclear transcription factor $\mathrm{Y}$ (NF-Y). As a result of UPR activation, genes involved in ER-associated protein degradation (ERAD) and redox homeostasis and chaperones are transcribed, in an attempt to preserve cell survival. In case of unresolvable stress, UPR shifts toward a proapoptotic program largely governed by CHOP that activates the apoptotic module by downregulation of B-cell lymphoma 2 (Bcl2) and upregulation of Bcl2-homologous (BH)-3-only proteins, and by upregulation of growth arrest and DNA damage-inducible protein (GADD34) that mediates elF2 $\alpha$ dephosphorylation resuming translation. CHOP is a shared target gene of all three arms of the UPR, but primarily induced by PERK branch. IRE1 $\alpha$ can also independently contribute to apoptosis by either sustaining its RIDD program or activation of c-Jun N-terminal kinase (JNK). ASK1, apoptosis signal-regulating kinase 1; ATF6, activating transcription factor 6; BH, Bcl2-homologous; BiP, glucose-regulated protein-78; elF2 $\alpha$, eukaryotic initiation factor 2; ER, endoplasmic reticulum; ERAD, ER-associated protein degradation; IRE1 $\alpha$, inositol-requiring enzyme 1 alpha; PERK, protein kinase R-like ER kinase; TRAF2, țumor necrosis factor receptor-âssociated factor 2; XBP1, X-box binding protein 1.

However, among the hallmarks of cancer, evasion from immune destruction and facilitation of protumorigenic inflammation are two of the most emergent themes of research addressing the role of ER stress or UPR in tumorigenesis over the last decade [19].

Almost all neoplasms undergo the process of cancer immunosurveillance before reaching an equilibrium marked by infiltration of mostly tumor-supporting immune cells, which favors the clonal expansion of cancer cells that have evolved the capability to escape immune control [20]. 


\section{Trends in Cancer}

Such a TME is characterized by T-cell exhaustion [resulting from chronic but suboptimal exposure to tumor-associated antigens (TAAs) in absence of costimulation and metabolic stress] or poor infiltration of cytotoxic $T$ cells, and presence of immunosuppressive immune cells (i.e., Type 2 polarized cells like $\mathrm{T}_{\mathrm{H}} 2 \mathrm{CD}^{+} \mathrm{T}$ cells, M2 macrophages, N2 neutrophils, or autoregulatory cells like $T_{\text {reg }}$ and myeloid-derived suppressor cells) [20,21]. This immune microenvironment fuels tumor growth because on the one hand it is incapable of killing the cancer cells (due to T-cell anergy), yet on the other it encourages either tolerance toward the tumor (through autoregulatory immune cells) or actively contributes to tumor progression via protumorigenic cytokines/chemokines (through Type 2 polarized or immunosuppressive immune cells).

In general, UPR activation in different cell types has been linked to inflammatory pathways through, for example, PERK-eukaryotic initiation factor 2 (elF2 $\alpha$ )-mediated inhibition of de novo synthesis, which by reducing the levels of the short-lived $I_{\kappa} B$ results in the activation of nuclear factor- $\mathrm{kB}(\mathrm{NF}-\kappa \mathrm{B})$ [22], the major regulator of inflammatory responses [23]. Likewise, the scaffolding function of IRE $1 \alpha$ can cause the tumor necrosis factor receptor-associated factor 2 (TRAF2)-mediated activation of $I_{\kappa} B$ kinase and apoptosis signal-regulating kinase 1-C-Jun Nterminal kinase (ASK1-JNK) signaling [24,25], while ATF6 may activate NF- $\mathrm{kB}$ through an AKTdependent pathway [26] (Figure 2). ER stress can trigger inflammatory responses also through the activation of the NLRP3 inflammasome that culminates in the production of interleukin-1 $\beta$ (IL-1 $\beta$ ) and IL-18, through both UPR-dependent and UPR-independent pathways $[27,28]$. Which of these UPR-modulated mechanisms prevalently operate in the cancer cells will likely depend on various intrinsic (oncogene expression, metabolic program, oxidative stress, etc.) and extrinsic TME factors (hypoxia, nutrient availability, etc.). However, once overactivated, these UPR-regulated pathways ultimately converge in the secretion of particular proinflammatory cytokines (e.g., IL-11, IL-1 $\beta, I L-6, I L-23$, and tumor necrosis factor $[23,29]$ ) in the extracellular milieu. On the one hand, these protumorigenic cytokines tend to promote cellular survival and engage the proliferation signaling cascade(s) in an autocrine/paracrine fashion, by binding their cognate receptors on cancer cell surface (which may also show upregulation, e.g., IL-6-based engagement of IL6R/gp130 on cancer cells [30]). On the other hand, these chemokines and cytokines can also recruit and/or sustain immunosuppressive immune cells to further fuel production of protumorigenic factors in a feed-forward loop [23]. While attracting regulatory or Type 2 polarized immune cells, cancer cells may also exploit UPR to blunt any de novo generation of antitumor immunity. For example, IL-23 secreted by tumor cells negatively influences the activity of $\mathrm{CD} 8^{+}$cytotoxic $T$ cells (cytotoxic $T$ lymphocytes) and supports the expansion of protumorigenic $T_{H} 17$ cells [31]. Through the activation of the NF-kB and p38 mitogen-activated protein kinases signaling pathways, ER stress can contribute to the upregulation of cyclooxygenase- 2 in the cancer cells and the release of prostaglandin $\mathrm{E}_{2}$, a negative regulator of antitumor immunity responses [32].

Moreover, activation of the UPR in cancer cells can propagate ER stress in myeloid cells, through a process termed 'transmissible ER stress'. This transmissible ER stress causes a proinflammatory/suppressive phenotype in myeloid cells, hallmarked by the upregulation of arginase and secretion of IL-6 and IL-23, thereby further compromising antitumor immunity responses [33]. Although the identity of the cancer cell-associated factors responsible for this crosstalk is still elusive, the enhanced proinflammatory responses induced in the receiver myeloid cells required a cancer cells-secreted Toll-like receptor 4 (TLR4) agonist [34].

The UPR can also modulate the presentation of antigens via the MHC molecule Class I, which is loaded with the antigenic peptide within the ER before being transported to the cell surface. However, how exactly ER stress affects this process in a tumoral context is still debated [35]. 


\section{Trends in Cancer}

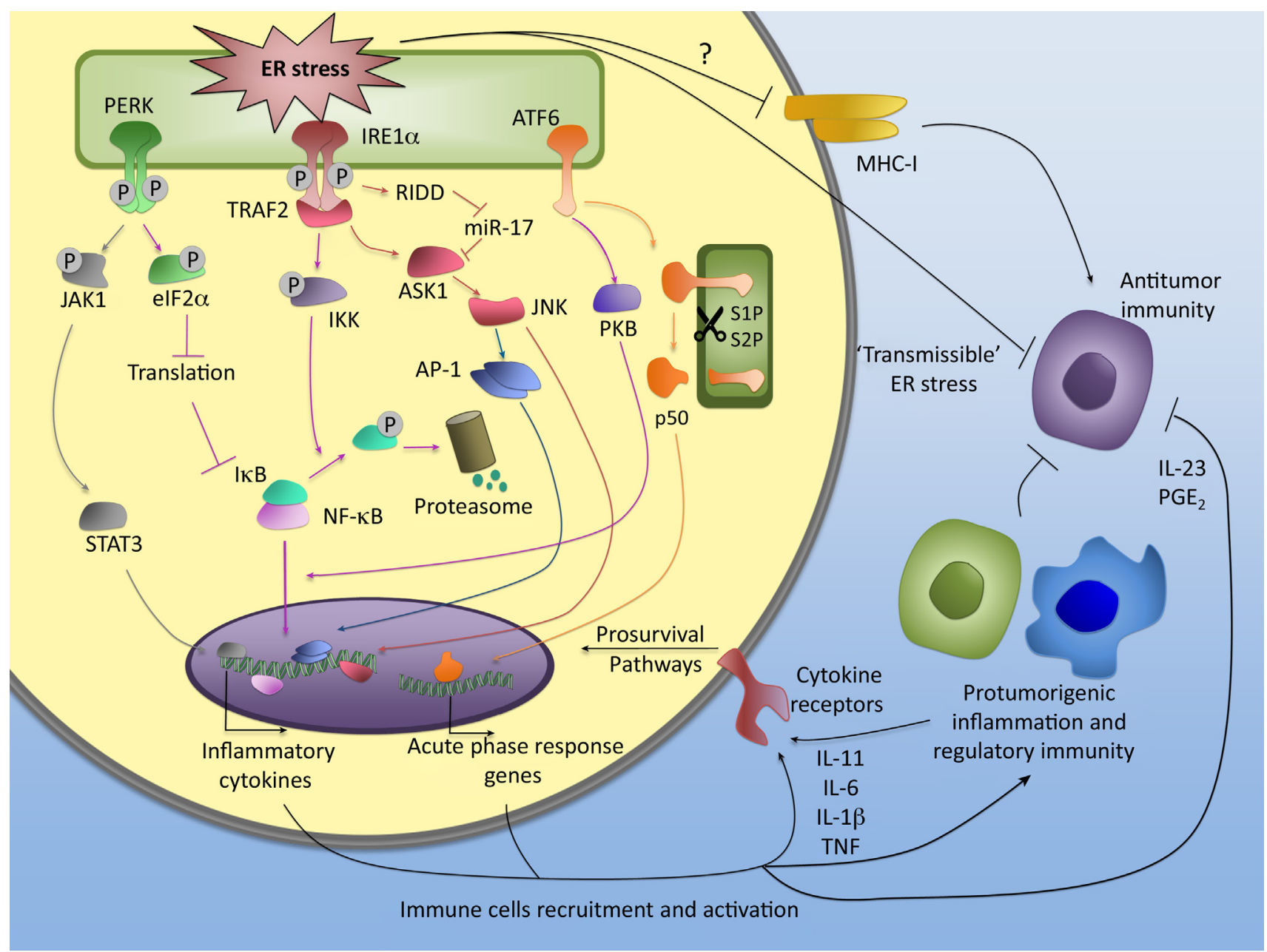

Figure 2. UPR-Related Proinflammatory Pathways and Immunologic Consequences. In case of ER stress, UPR sensors may engage proinflammatory pathways. Indeed, by means of translation attenuation, PERK promotes the stoichiometry in favor NF-kB with respect to its inhibitor I $\mathrm{kB}$. PERK has been recently linked also to the activation of STAT3 through phosphorylation of Janus kinase 1 (JAK1) [84]. Besides signaling through XBP1s, IRE1 $\alpha$ can exert a scaffolding function leading to the assembly of a protein platform called UPRosome that comprises different adaptor and regulatory proteins, such as tumor necrosis factor receptor-associated factor 2 (TRAF2), involved in proinflammatory processes. TRAF2 can cause the recruitment and activation of $\mid \kappa B$ kinase (IKK) that in turn lead to the degradation of I $\mathrm{kB}$ through phosphorylation, freeing NF-KB. IRE1 $\alpha$ can also activate apoptosis signal-regulating kinase 1 (ASK1) by the RIDD-mediated degradation of miR-17, through either direct [85] or indirect mechanisms [86], leading in turn to the activation of JNK. JNK can itself transcriptionally regulate many inflammatory genes or activate activator protein 1 (AP-1), another potent proinflammatory transcription factor. AP-1 is a dimeric complex and its subunit composition dictates the target genes, with a variable outcome ranging from protumorigenic to antitumorigenic. ATF6 may also control NF-kB through protein kinase B (PKB), but its key contribution to the inflammatory milieu is through the induction of an acute phase response that leads to the secretion of serum proteins involved in inflammation. The proinflammatory cytokines released may act in an autocrine manner or recruit immune cells (i) to support the proinflammatory environment, activating prosurvival pathways in the cancer cell, or (ii) to suppress active antitumor immunity upon polarization toward Type 2 phenotype. At the same time, cancer cells inhibit antitumor immunity by decreasing antigen presentation and by transmissible ER stress. ATF6, activating transcription factor 6; ER, endoplasmic reticulum; IRE1 $\alpha$, inositol-requiring enzyme 1 alpha; JNK, c-Jun N-terminal kinase; IL, interleukin; NF-кB, nuclear factor кB; PERK, protein kinase R-like ER kinase; $\mathrm{PGE}_{2}$, prostaglandin $\mathrm{E}_{2}$; RIDD, regulated IRE1 $\alpha$-dependent decay; STAT3, signal transducer and activator of transcription 3; UPR, unfolded protein response; XBP1, X-box binding protein 1.

Altogether, these studies indicate that the initial aim of ER stress response is to repair the tumor-inflicted tissue 'wounding' by attraction of innate immune cells (e.g., neutrophils, monocytes, mast cells) that exert potent antimicrobial (and in this case tumor cytotoxic) functions by producing high levels of reactive oxygen species (ROS) and inflammatory cytokines and favor new tissue formation by releasing proangiogenic factors and metalloproteinases. Yet, chronic continuation of such responses ultimately fuels autoregulatory inflammatory 


\section{Trends in Cancer}

loops and degradation of tumor stroma supporting tumor migration, eventually favoring (rather than impede) tumorigenesis [36].

\section{UPR and ER Stress-Driven Immunogenic Cancer Cell Death}

The chronic persistence of mild ER stress modulates the extracellular microenvironment to support tumorigenesis. However, the overall situation changes when ER stress is exceedingly elevated and UPR is skewed toward the proapoptotic module. Indeed, in this situation, the ER may communicate the danger state of a stressed cell to the microenvironment eventually orchestrating an antitumor immune response. Apoptosis has been traditionally documented to be an immunologically silent or tolerogenic process (and hence referred to as 'physiological apoptosis'), while necrosis has been described as a proinflammatory cell death pathway that facilitates protumorigenic processes [37]. Therefore, since most of the anticancer therapies currently used in the clinic primarily induce a mixture of physiological apoptosis and necrosis (depending on the dosage and number of cycles administered), they were all thought to facilitate either tolerogenicity toward dying cancer cells or protumorigenic inflammation [38]. However, during the last decade, this dogmatic view has changed. Indeed, several independent studies have identified that a particular, yet diverse set of commonly used anticancer therapies (such as anthracyclines, radiotherapy, oncolytic viruses, photodynamic therapies) can accentuate the immunogenic potential of dying cancer cells. A distinctive structurefunction relationship for different ICD inducers is virtually nonexistent, as exemplified by the different immunogenic potential of two structurally related compounds like oxaliplatin and cisplatin. The key to ICD induction for all these inducers is the concomitant and sustained induction of ROS and ER stress [39]. In fact, ER stress is so crucial for the paradigm that different ICD inducers may be classified based on the quality of ER stress they induce (e.g., Type I or Type II inducers of ICD, discussed later). Interestingly, during ICD, while all three branches of the UPR are activated, yet only the PERK branch is so far mandatory for ICD. ICDinducing anticancer treatments elicit a form of ER stress that is capable of warning the immune cells of a state of danger, through the release (or surface exposure) of immunostimulatory factors or damage-associated molecular patterns (DAMPs) acting as danger signals. DAMPs are endogenous molecules with various housekeeping functions, which acquire immunomodulatory functions when released or surface exposed by the dying cancer cells. In analogy to the pathogen-associated molecular patterns, DAMPs bind to pattern recognition receptors (including various TLRs) on the innate immune cells [e.g., professional antigen-presenting cells, like dendritic cells (DCs)] and favor the establishment of a productive interface between the dying cancer cells and the immune system (Figure 3). This, in turn, results in the priming of the host immune system for the TAAs and subsequent elicitation of TAA-specific T-cell-mediated immune responses, leading to the elimination of the residual cancer cells as well as the establishment of an immunological memory [40].

There are specific DAMPs associated with ICD: (i) early surface exposure (ecto-) of the ER chaperone calreticulin (CRT) during the preapoptotic phase [41], wherein ecto-CRT acts as an 'eat-me' signal and is recognized by the CD91 receptor on phagocytes, stimulating the engulfment of dying cancer cells. Of note, other ER or cytosolic chaperones like heat shock protein (HSP)-70/90 can also be surface exposed and may act, redundantly with ecto-CRT, as 'eat me' signals [42]; (ii) active or passive secretion of ATP [43] during the preapoptotic or early/ midapoptotic phases that could act either as a potent short-range 'find-me' signal for attracting monocytes by binding the $\mathrm{P}_{2} \mathrm{Y}_{2}$ purinoceptors, or as a proinflammatory (inflammasomeactivating) molecule by binding the $\mathrm{P}_{2} \mathrm{X}_{7}$ receptor on innate immune cells; (iii) passive, lateapoptotic release of high mobility group box 1 (HMGB1) that binds TLR2 and TLR4 on DCs, stimulating production of proinflammatory cytokines and assisting in proper antigen presentation [44]; and (iv) passive release of nucleic acids, including double-stranded DNA, that signal via TLR-7/8/9 on innate immune cells (like neutrophils), thereby regulating their activation and 


\section{Trends in Cancer}

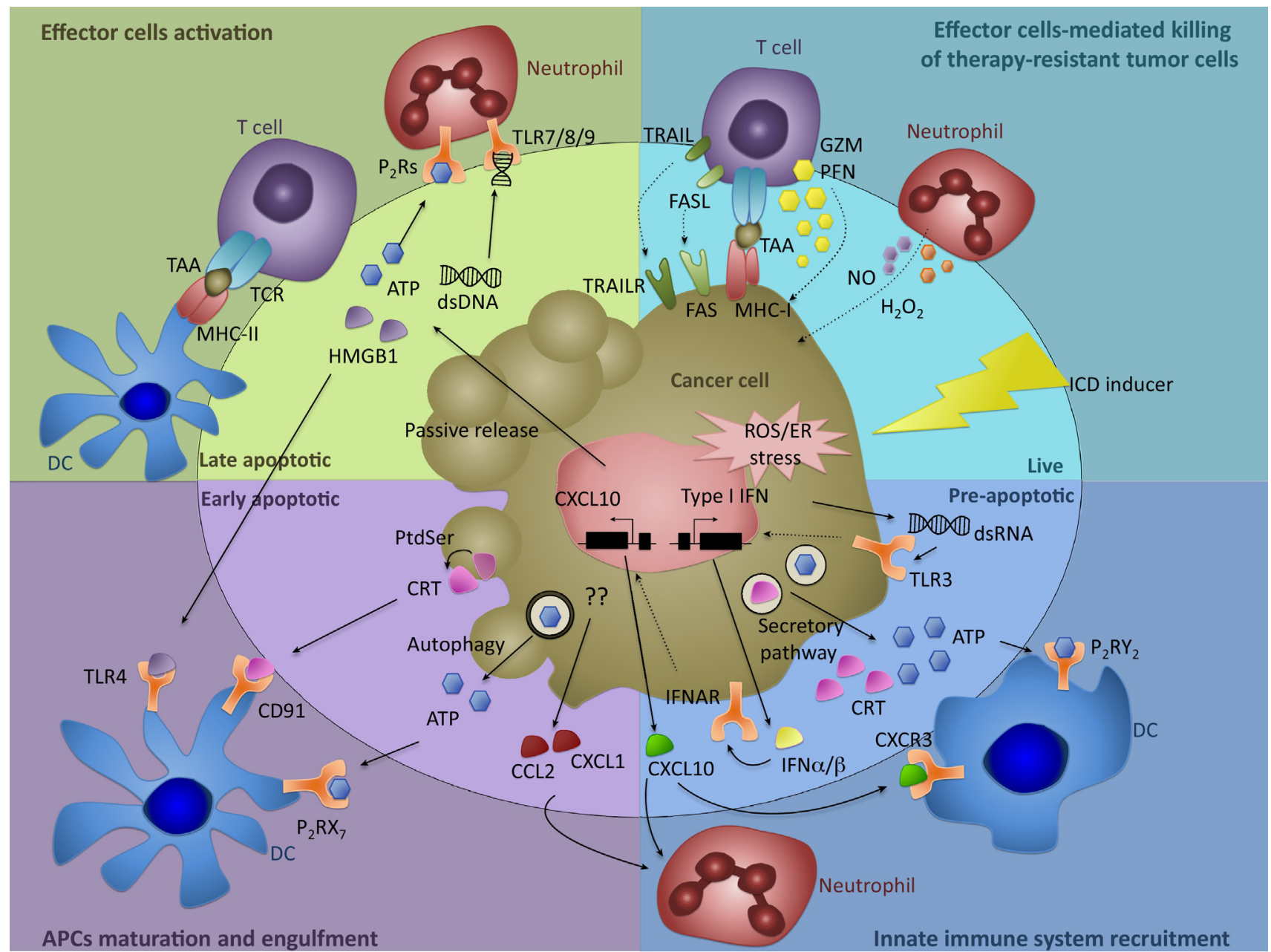

Trends in Cancer

Figure 3. Immunogenic Cell Death (ICD). Upon treatment with ICD inducers, cancer cells rapidly mobilize the ER luminal chaperone CRT to the plasma membrane through a pathway that requires PERK modulation of the secretory pathway. Alternatively, CRT could be exposed in association with phosphatidyl-serine (PtdSer) when the latter flips toward the outer side of the plasma membrane. ATP instead may be released either by secretory pathway (preapoptotic phase) or by autophagy (early apoptotic phase). In the late apoptotic phase, HMGB1, ATP, and dsDNA diffuse passively toward the extracellular space because of plasma membrane permeabilization. ICD inducers stimulate the cell to produce Type I IFNs that act via an autocrine signaling pathway through the IFN receptor (IFNAR), leading to the production and secretion of CXCL10. ATP and CXCL10 instead act as find-me signals to recruit DCs. ATP. HMGB1, and dsDNA lead to DCs maturation and activation, whereas CRT favors the engulfment of cancer cells. Once activated, DCs cross-present cancer cell-associated antigens (TAA) to $C D 4^{+}$and $C D 8^{+} T$ cells that after clonal proliferation mediate the killing of live residual tumor cells through extrinsic cell death pathways [e.g., granzyme (GZM)-perforin (PRF) or FasL/TRAlL pathways]. Recently, it has been described that CXCL1 and CCL2 (released by the cancer cell through a yet unidentified mechanism) together with CXCL10 recruit neutrophils, whose activation is mediated by ATP and dsDNA. Activated neutrophils support the killing of therapy-resistant cancer cells through respiratory burst mediated by nitric oxide (NO) and hydrogen peroxide $\left(\mathrm{H}_{2} \mathrm{O}_{2}\right)$. APC, antigen-presenting cell; CCL2, chemokine (C-C motif) ligand 2; CRT, calreticulin; CXCL10, cancer cell-autonomous release of chemokine (C-X-C motif) ligand 10; DC, dendritic cell; dsDNA, double-stranded DNA; ER, endoplasmic reticulum; HMGB1, high mobility group box 1; ICD, immunogenic cancer cell death; IFN, interferon; IFNAR, interferon- $\alpha / \beta$ receptor; PERK, protein kinase R-like ER kinase; TAA, tumor-associated antigen; TCR, T-cell receptor; TLR, Toll-like receptor; TRAIL, tumor necrosis factor-related apoptosis inducing ligand.

anticancer activity [45]. Of note, cells undergoing ICD can also release Annexin A1 that binds to formyl peptide receptor-1/2 (FPR1 or FPR2) on innate immune cells. However, the immunological impact of Annexin A1 is controversial since one study documented a proimmunogenic effect centered on DCs [46], while another documented an immunosuppressive effect centered on neutrophils [45]. 


\section{Trends in Cancer}

Beyond emission of DAMPs/danger signals, the cancer cells undergoing ICD can also activate the immune system by performing 'altered-self mimicry', that is, mimicking host defense response typically triggered by pathogens or viral infection, which stimulates strong immunological responses against the 'altered-self' cell (e.g., cells undergoing ICD). For instance, doxorubicin-induced ICD has been shown to be associated with the release of dsRNA that binds to TLR3 on other cancer cells (paracrine signaling), thereby inducing release of Type I interferon (IFN) cytokines [47], which mediate anticancer immunity. By contrast, cancer cells dying via ICD can also orchestrate a cancer cell-autonomous release of chemokine ( $\mathrm{C}-\mathrm{X}-\mathrm{C}$ motif) ligand 1 (CXCL1), chemokine (C-C motif) ligand 2 (CCL2), and CXCL10 that attract neutrophils and eventually pave the way for their ATP- and nucleic acids-based activation, in a manner reminiscent of response to bacterial or viral infections. These neutrophils eventually exert direct anticancer cytotoxic activity against residual cancer cells via respiratory burst [45]. However, there is a significant inducer- and context-dependent plasticity in the amount, diversity, kinetics, and immunological activity of DAMPs, danger signals, and cytokines/chemokines emitted upon treatment (Table 1) [48,49]. At the same time, many non-ICD inducers have been observed to elicit mobilization of one or more DAMPs [49], although without promoting effective DC maturation and antitumor immunity.

Table 1. Characteristics of Clinically Relevant ICD Inducers

\begin{tabular}{|c|c|c|c|c|c|}
\hline ICD inducer & Indications & On-target effect & ER effect & DAMPs & Note \\
\hline \multicolumn{6}{|l|}{ Type I ICD inducers } \\
\hline Bleomycin & $\begin{array}{l}\text { Multiple } \\
\text { hematological and } \\
\text { solid tumors }\end{array}$ & $\begin{array}{l}\text { DNA-damaging } \\
\text { agent }\end{array}$ & $\begin{array}{l}\text { ER stress induced through an } \\
\text { unknown mechanism }\end{array}$ & $\begin{array}{l}\text { CRT, ATP, } \\
\text { IFN, HMGB1 }\end{array}$ & $\begin{array}{l}\text { Ambivalent immunological } \\
\text { outcome due to expansion } \\
\text { of } T_{\text {reg }} \text { cells }\end{array}$ \\
\hline Bortezomib & $\begin{array}{l}\text { Mantle cell } \\
\text { lymphoma } \\
\text { Multiple myeloma }\end{array}$ & $\begin{array}{l}\text { Proteasomal } \\
\text { inhibitor }\end{array}$ & $\begin{array}{l}\text { ER stress induced indirectly by } \\
\text { protein overload due to impaired } \\
\text { ER-associated degradation } \\
\text { machinery }\end{array}$ & $\begin{array}{l}\text { CRT, HSP70, } \\
\text { HSP90, IFN, } \\
\text { HMGB1 }\end{array}$ & \\
\hline Cyclophosphamide & $\begin{array}{l}\text { Multiple } \\
\text { hematological and } \\
\text { solid tumors }\end{array}$ & Alkylating agent & $\begin{array}{l}\text { ER stress induced through } \\
\text { an unknown mechanism }\end{array}$ & $\begin{array}{l}\text { CRT, ATP, } \\
\text { IFN, HMGB1 }\end{array}$ & $\begin{array}{l}\text { Immunosuppressive at } \\
\text { high doses, decreases } \\
\text { the percentages of } T_{\text {reg }} \\
\text { at lower or metronomic } \\
\text { doses }\end{array}$ \\
\hline $\begin{array}{l}\text { Anthracyclines } \\
\text { (doxorubicin, epirubicin, } \\
\text { idarubicin, mitoxantrone) }\end{array}$ & $\begin{array}{l}\text { Multiple } \\
\text { hematological and } \\
\text { solid tumors }\end{array}$ & $\begin{array}{l}\text { DNA- } \\
\text { intercalating } \\
\text { agent }\end{array}$ & $\begin{array}{l}\text { Associate with cellular } \\
\text { membranes (including ER) } \\
\text { and cause ROS production } \\
\text { resulting in ROS-based ER } \\
\text { stress }\end{array}$ & $\begin{array}{l}\text { CRT, ATP, IFN, } \\
\text { HSP70, HMGB1 }\end{array}$ & \\
\hline Oxaliplatin & Colorectal carcinoma & $\begin{array}{l}\text { Alkylating } \\
\text { agent }\end{array}$ & $\begin{array}{l}\text { Associates with cellular } \\
\text { membranes (including ER) } \\
\text { and causes ROS production } \\
\text { resulting in ROS-based ER } \\
\text { stress }\end{array}$ & $\begin{array}{l}\text { CRT, ATP, IFN, } \\
\text { HSP70, HMGB1 }\end{array}$ & \\
\hline \multicolumn{6}{|l|}{ Type II ICD inducers } \\
\hline $\begin{array}{l}\text { Hypericin-photodynamic } \\
\text { therapy }\end{array}$ & $\begin{array}{l}\text { Nonmelanoma skin } \\
\text { cancer, cutaneous } \\
\text { T-cell lymphoma, } \\
\text { mesothelioma, } \\
\text { basal or squamous } \\
\text { cell carcinoma }\end{array}$ & $\begin{array}{l}\text { Induction of } \\
\text { oxidative stress } \\
\text { with damage } \\
\text { to ER membranes }\end{array}$ & $\begin{array}{l}\text { ER membrane-associated } \\
\text { photosensitive drug causes } \\
\text { massive production of ROS } \\
\text { and ROS-based ER stress } \\
\text { upon light activation }\end{array}$ & $\begin{array}{l}\text { CRT, ATP, HSP70, } \\
\text { HSP90, HMGB1 }\end{array}$ & \\
\hline Oncolytic viruses & $\begin{array}{l}\text { In clinical trials for } \\
\text { multiple solid } \\
\text { cancers }\end{array}$ & $\begin{array}{l}\text { Selective lysis of } \\
\text { malignant cells }\end{array}$ & $\begin{array}{l}\text { ER stress induced through } \\
\text { viral protein overload }\end{array}$ & & \\
\hline
\end{tabular}




\section{Trends in Cancer}

Either mRNA and/or protein level of DAMPs (together with markers of ER stress) has been proposed as possible biomarkers for ICD induction in patients. However, their prognostic value shows significant variability according to different studies performed in various cancer types, possibly due also to technical and conceptual limits (Table 1).

However, while ROS-mediated ER stress is a central process conferring to apoptotic cell death an immunogenic character, ER stress may be dispensable for ICD signaling and antitumor immunity elicited by other forms of regulated cell death, such as necroptosis. Indeed, a recent study showed that cancer cells induced to undergo necroptosis, via Fas-associated protein with death domain (FADD) dimerization-mediated receptor-interacting serine/threonine-protein kinase 3 (RIPK3) activation, released some of ICD-related DAMPs (i.e., ATP and HMGB1) and triggered adaptive immune responses in vivo without an apparent contribution of ER stress [50]. In the context of chemotherapy-induced ICD, the necroptotic mediators RIPK3 and mixed lineage kinase domain-like protein (MLKL) were shown to contribute to immunogenicity and DAMPs emission, but whether chemotherapy-induced ER stress was required has not been evaluated [51]. Finally, it would be important to evaluate immunogenic potential of other forms of therapy-induced regulated necrosis, such as ferroptosis, distinguished by elevated ER stress and ROS levels.

Thus, while cancer cell-constitutive activation of the prosurvival program of the UPR facilitates many aspects of the protumorigenic microenvironment, harnessing the lethal arm of the UPR through oxidative stress enables the release of cancer cell death-associated danger signals, which are decoded by the immune system and escalate antitumor immune responses.

\section{ER Stress-Driven Pathways during ICD in Cancer Cells}

Considering the compelling reliance of ICD on the induction of a sustained ER stress, it is not surprising that the different intensity and the kinetics of ER stress may have a strong impact on the quality and quantity of danger signaling. This led to the subdivision of ICD inducers into two broad categories (Table 1): Type I and Type II ICD inducers [40]. 'Type I ICD inducers' encompass all the drugs that trigger ICD-associated immunogenicity through secondary 'off-target' (mostly mild) ER stress in parallel with the main 'on-target' effect that drives apoptosis via non-ER targets. Most clinically employed ICD inducers, such as anthracyclines, oxaliplatin, bortezomib, cyclophosphamide, and radiotherapy, fall within this category [48]. 'Type II ICD inducers', instead, selectively target the ER and orchestrate both danger and apoptotic signaling through 'focused/on-target' (ROS-based) ER stress. The first treatment identified as Type II ICD inducer was hypericin (Hyp)-based photodynamic therapy [52], followed by identification of other treatments, such as some oncolytic viruses (e.g., Newcastle disease virus) $[53,54]$ and platinum" $-N$-heterocyclic carbene complex [55]. The quality and quantity of ER stress elicited by the Type II ICD inducers are superior to Type I ICD inducers in terms of both overall amounts and speed of the emission of danger signals/DAMPs exposed during the preapoptotic stage [40]. Irrespective of this, although the signaling pathways leading to DAMP exposure/secretion show a certain degree of dissimilarity between Type I and Type II ICD inducers (Figure 4), they both share key apical and proximal mediators and require an intact secretory pathway and the actin cytoskeleton.

As presented in Figure 4, PERK is at the 'core' of ICD and the apical coordinator of DAMP trafficking mechanisms. Intriguingly, the function of PERK seems to differ between Type I (activating classical UPR pathway through elF2 $\alpha$ phosphorylation) and Type II (regulating expansion of the secretory pathway through an as-yet-unknown interactor) ICD inducers. Recently, a peculiar ICD inducer at the interface between Type I and Type II, high hydrostatic pressure, has also been shown to engage a PERK-modulated pathway for ecto-CRT exposure involving caspase-2 [56]. 


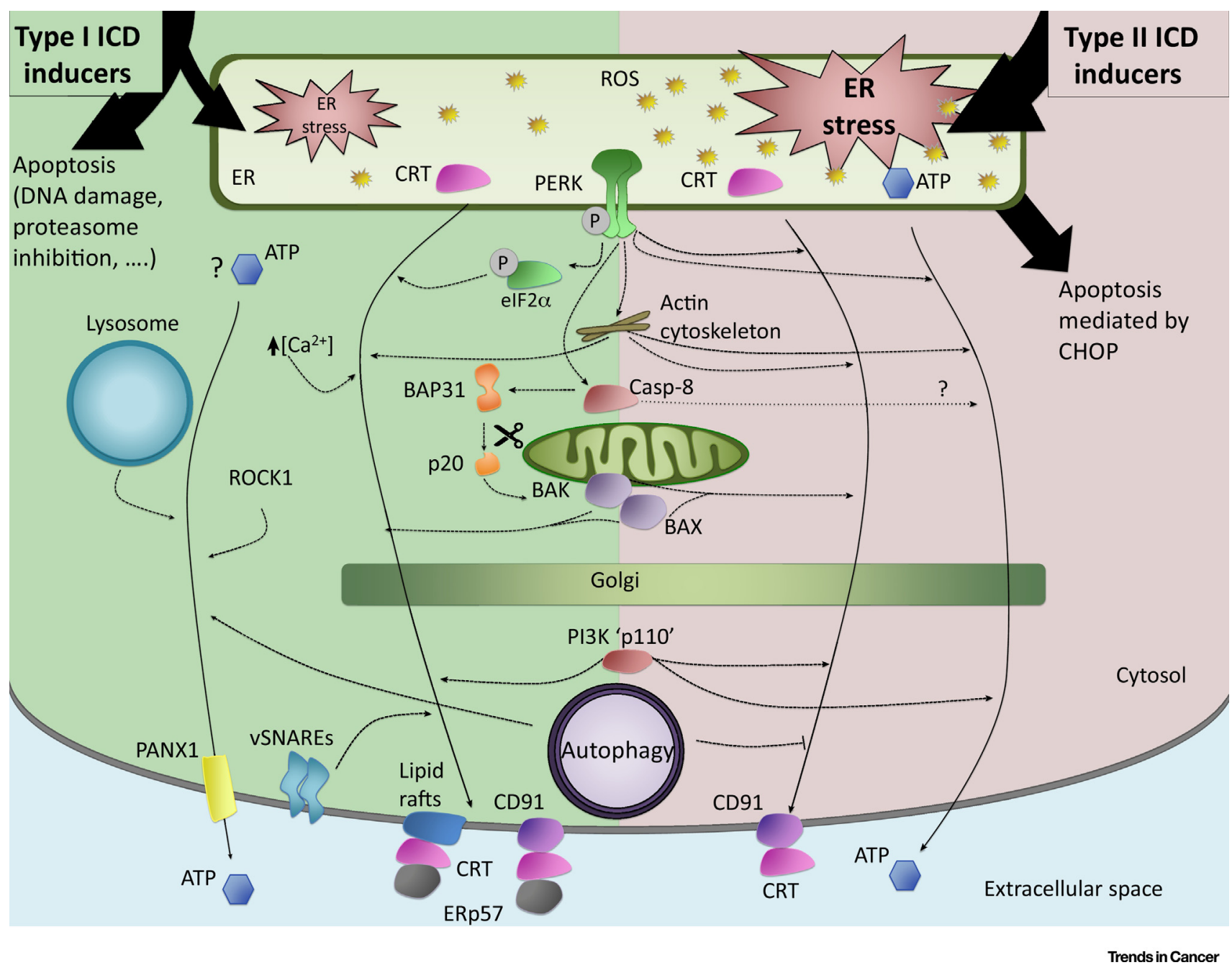

Figure 4. DAMPs Mobilization Pathways of Type I versus Type II ICD Inducers. Type I and Type II ICD inducers are defined based on their side or focused effect on the ER, respectively. Their different mode of action is reflected on several differences in the pathways engaged for DAMPs translocation. Surface exposure of CRT upon treatment with Type I ICD inducers requires a rather complex pathway with concomitant activation of three modules: (i) the ER stress-ROS signaling mediated by the activation of the PERK-elF2 $\alpha$ axis; (ii) the apoptotic signaling, reliant on the preapoptotic caspase-8-mediated cleavage of B-cell receptor-associated protein 31 (BAP31) and regulated by Bax/Bak and $\mathrm{Ca}^{2+}$ signals; and (iii) a soluble $\mathrm{N}$-ethylmaleimide-sensitive factor activating protein receptor (SNARE)-dependent, ERto-Golgi anterograde secretory pathway [58]. Instead, the pathway employed upon treatment with Type II ICD inducers is simpler and relies only on PERK, Bax, Bak, and the secretory pathway [52]. Surface exposure of CRT occurs in association with ERp57 by binding to lipid rafts or low-density lipoprotein receptor-related protein 1 (LRP1) upon treatment with Type I ICD inducers, whereas only CD91 is necessary for Type II inducers. Moreover, Type II ICD-induced ATP secretion follows a pathway highly overlapping with ecto-CRT except for Bax/Bak [52] and involving partially caspase 8, whereas Type I ICD inducers have been demonstrated to require an independent pathway mediated by autophagy [87]. CRT, calreticulin; DAMPs, damage-associated molecular patterns; elF2 $\alpha$, eukaryotic initiation factor 2; ER, endoplasmic reticulum; ICD, immunogenic cancer cell death; PANX1, Pannexin 1; PERK, protein kinase R-like ER kinase; PI3K, phosphatidylinositol-4,5-bisphosphate 3-kinase; ROCK1, rho-associated, coiled-coil-containing protein kinase 1; ROS, reactive oxygen species; SNARE, SNAP (soluble N-ethylmaleimide-sensitive factor attachment protein) receptor.

The reason behind the reliance of the danger signaling on PERK rather than on other UPR sensors (e.g., IRE1 $\alpha$ ) remains enigmatic. Likewise, the mechanism, shared by Type I and Type II ICD inducers, linking PERK to intracellular $\mathrm{Ca}^{2+}$ elevation and the actin cytoskeleton, in the path to mobilize DAMPs, remains unclear. This discrepancy could be explained by a newly discovered, UPR-independent function of PERK in modulating the dynamics of the actin cytoskeleton, through its interaction with the actin-binding protein Filamin A (FLNA) [57]. A recent study from our laboratory has provided compelling evidence showing that PERK is able to sense and rapidly respond to cytosolic $\mathrm{Ca}^{2+}$ elevations through its cytosolic domain, by 


\section{Trends in Cancer}

enabling the formation of ER-plasma membrane (PM) appositions, resulting in opening of the STIM1-ORA1 sensor stromal interaction molecule 1 (STIM1)-ORAI calcium release-activated calcium modulator 1 (ORAl1) channel and $\mathrm{Ca}^{2+}$ influx, through its interaction with FLNA [57]. This newly identified function of PERK in forming ER-PM contact sites could be relevant for the mechanisms of trafficking of DAMPs via the secretory pathway and SNAP (soluble N-ethylmaleimide-sensitive factor attachment protein) receptor (SNARE)-mediated exocytosis, housekeeping processes that have been shown to be required for Type I and Type II ICD inducers $[52,58]$. A PERK-FLNA axis could in fact sustain, through the rapid formation of ER-PM junctions, intracellular $\mathrm{Ca}^{2+}$ levels and $\mathrm{Ca}^{2+}$-modulated actin cytoskeleton remodeling, which are key mediators of ER-to-Golgi trafficking and vesicle exocytosis [59].

Overall, the key function of the UPR and ER stress sensors in modulating ICD immunogenicity by guiding the surface exposure and release of DAMPs and the master role of PERK in this process have been thoroughly demonstrated. However, more studies are still needed to unravel key mechanisms underlying ICD induction that are common to, if possible, all ICD inducers.

\section{Clinical Implications of ER Stress-Driven Immunogenicity for Cancer Immunotherapy}

Most of the currently identified ICD inducers have been intensively used in clinical practice and often (but not always) constitute the first line of therapy (especially when employed through 'onlabel' use, Table 1) [60]. However, the current (direct) evidence for ICD is largely derived from rodent studies (Box 1), whereas clinical evidence is still limited. Indeed, highly reliable biomarkers for monitoring ICD induction in situ are still missing (Table 2). Nevertheless, retrospective analyses support (to a certain extent) the existence of ICD in the clinical settings, based on the correlation between the expression levels of key ICD-related genes and a more favorable prognosis selectively in patients treated with ICD inducers [61,62]. Similarly, mutations or defects in the molecular machinery mediating ICD are usually associated with worse prognosis in patients treated with ICD inducers [43,63-65].

However, the characterization of ICD in the clinic is complicated due to (i) UPR-driven protumorigenic pathways present in a clinically progressive tumor; and (ii) the relatively immunocompromised status or low immunological fitness of the patients (e.g., inactivating polymorphisms in immunoreceptors like TLRs or immunomodulators like IFNs). Moreover, cancer cells may antagonize the prophagocytic activity of therapy-induced ecto-CRT by upregulation of 'don't eat-me' signals such as CD47, whose neutralization would be desirable through the use of anti-CD47 antibody that has shown promising antitumorigenic effects [66] (Figure 5, Key Figure).

Box 1. Experimental Models to Evaluate ICD

Several biochemical assays have been developed in the last decade to measure induction of UPR, surface exposure of ER/cytosolic chaperones CRT, HSP70 and HSP90, and release of ATP or HMGB1 as markers of ICD induction [88]. However, none of these assays, even in combination, could discriminate with certainty between ICD and non-ICD inducers and this highlights a need for investigating new determinants of immunogenicity. Therefore, ICD evaluation still requires systematic analysis of the generation of an antitumor adaptive immune response. This could be partially evaluated in vitro in coculture assays of treated cancer cells with various immune cells; however, these are done with an often unconfirmed assumption that such immune cells would be chemoattracted in those combinations in a treated tumor in vivo. Because of this, the gold standard for determining ICD induction consists of prophylactic immunization. This entails vaccination of immunocompetent syngeneic mice with dying cancer cells, followed by a rechallenge 1 week later with the same, but live, cancer cells. The percentage of mice that eradicated the tumor give an estimation of the ICD-inducing potential of an anticancer therapy tested in the vaccination step. However, this approach is significantly different from the clinical practice, where therapy is administered after the tumor has manifested (and in many cases, undergone surgical resection). Another approach consists of systemic or intratumoral administration of the therapeutics in both immunosuppressed and immunocompetent mice, and any antitumor efficacy in the latter is a sign of adaptive immune system-dependent immunogenic potential of a therapy. 


\section{Trends in Cancer}

Table 2. Clinical Biomarkers of ICD

\begin{tabular}{|c|c|c|c|c|c|}
\hline ICD biomarker & Cancer type & ICD treatment & Detection method & $\begin{array}{l}\text { Prognostic } \\
\text { value }\end{array}$ & Refs \\
\hline \multirow[t]{5}{*}{ HMGB1 } & Breast cancer & Anthracyclines & $\begin{array}{l}\text { Intratumoral nuclear HMGB1 } \\
\text { [immunohistochemistry }(\mathrm{IHC})]\end{array}$ & Good & [75] \\
\hline & $\begin{array}{l}\text { Squamous cell } \\
\text { carcinoma }\end{array}$ & Chemo/radiotherapy & Intratumoral (IHC) and serum (ELISA) & Good & [76] \\
\hline & $\begin{array}{l}\text { Non-Hodgkin } \\
\text { lymphoma }\end{array}$ & $\begin{array}{l}\text { Vaccination with DCs loaded ex vivo with } \\
\text { heat-shock-, } \gamma \text {-irradiation- and ultraviolet } \\
\text { C rays-treated cancer cells }\end{array}$ & Ex vivo, extracellular (ELISA) & Neutral & [73] \\
\hline & Advanced solid tumors & Oncolytic adenoviruses & Serum (ELISA) & $\mathrm{Bad}$ & [77] \\
\hline & \multicolumn{5}{|c|}{$\begin{array}{l}\text { - The oxidative status of HMGB1 determines its immunomodulatory activity, from immunostimulatory to immunosuppressive. } \\
\text { - HMGB1 may be released from the necrotic center of the tumor, acting as marker of tumor burden. } \\
\text { - High HMGB1 baseline could indicate preoccurred recognition of the tumor by the immune cells coupled to } \\
\text { active immunosuppression to sustain immune escape. }\end{array}$} \\
\hline \multirow[t]{6}{*}{ CRT } & $\begin{array}{l}\text { Non-Hodgkin } \\
\text { lymphoma }\end{array}$ & $\begin{array}{l}\text { Vaccination with DCs loaded ex vivo with } \\
\text { heat-shock-, } \gamma \text {-irradiation- and ultraviolet } \\
\text { C rays-treated cancer cells }\end{array}$ & $\begin{array}{l}\text { Ex vivo, surface exposed on tumor } \\
\text { cells (flow cytometry) }\end{array}$ & Good & [73] \\
\hline & Ovarian cancer & Paclitaxel & Bulk tumor (microarray) & Good & [64] \\
\hline & Lung cancer & Radiotherapy & Bulk tumor (microarray) & Good & [64] \\
\hline & Acute myeloid leukemia & Anthracyclines & Surface exposed (flow cytometry) & Neutral & [78] \\
\hline & Colorectal cancer & 5-Fluorouracil & Intratumor $(\mathrm{IHC})$ & Good & [79] \\
\hline & \multicolumn{5}{|c|}{ - Difficulty to differentiate between intracellular and surface-exposed calreticulin. } \\
\hline \multirow[t]{2}{*}{ ER stress $(\mathrm{elF} 2 \alpha)$} & Breast cancer & Anthracyclines & Intratumor phosphorylation status $(\mathrm{IHC})$ & $\mathrm{Bad}$ & [80] \\
\hline & \multicolumn{5}{|c|}{ - elF2 $\alpha$ is required only for Type I ICD inducers. } \\
\hline ER stress (XBP1) & Acute myeloid leukemia & Anthracyclines & Spliced XBP1 in tumor cells (PCR) & Good & [81] \\
\hline ER stress (BiP) & $\begin{array}{l}\text { Non-Hodgkin } \\
\text { lymphoma }\end{array}$ & Bortezomib & Intratumor $(\mathrm{IHC})$ & $\mathrm{Bad}$ & [82] \\
\hline IFN & Breast cancer & Anthracyclines & $\begin{array}{l}\text { Intratumor MX1 metagene (microarray), } \\
M X 1 \text { and TLR3 (IHC) }\end{array}$ & Good & [47] \\
\hline
\end{tabular}

By contrast, it is unlikely that the induction of ICD alone can completely subvert the highly immunosuppressive TME. Thus, a combinatorial approach may result in improved therapeutic outcomes. To accomplish this, it would be advantageous to combine ICD inducers (e.g., radiotherapy or anthracyclines) with immune-checkpoint blockers (ICBs). Immune checkpoints are a set of inhibitory ligands that are positioned between the tumor/stromal cells and tumorspecific T cells to prevent their antitumoral cytotoxic activity. By blocking immune checkpoints with ICBs [e.g., targeting cytotoxic T-lymphocyte-associated protein-4 (CTLA-4), programmed cell death-1 (PD-1) and others under clinical evaluation], the negative checkpoint is withdrawn and $T$ cells are able to exert their antitumor activity. This combination could result in bidirectional synergy, since the efficacy of ICBs tends to be limited to cancer patients exhibiting high tumoral mutational load and/or gene copy-number alterations and high tumoral pre-existing/basal Tcell infiltrates (i.e., 'T cell-hot' tumors) $[67,68]$. Thus, the induction of ICD could facilitate an initial T-cell-driven anticancer immunity, by resetting the TME favoring infiltration of $\mathrm{T}$ cells skewed toward a $T_{H} 1$-mediated immunity, which could be then sustained by the presence of ICBs (Figure 5) [69]. Of note, such combinatorial approaches have been demonstrated in preclinical models [70] to have superior benefit, and the results from already ongoing clinical trials (especially combining radiotherapy with ICBs in nonsmall cell lung cancer patients) will be essential to confirm it. 


\section{Trends in Cancer}

(C)

$$
\text { ICD + Adoptive T cell transfer }
$$

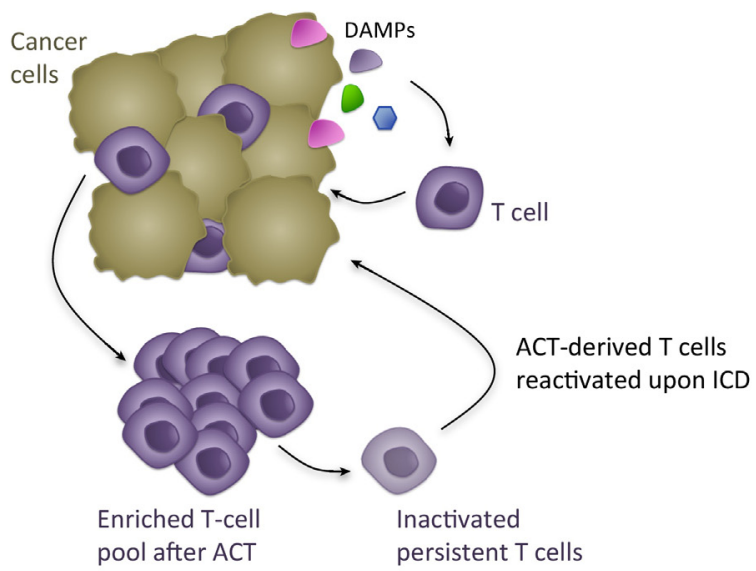

(B)

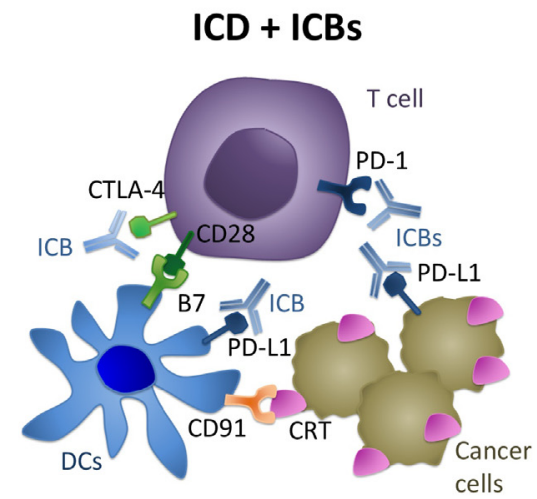

(D)

\section{Autologous DC vaccine + ICD}

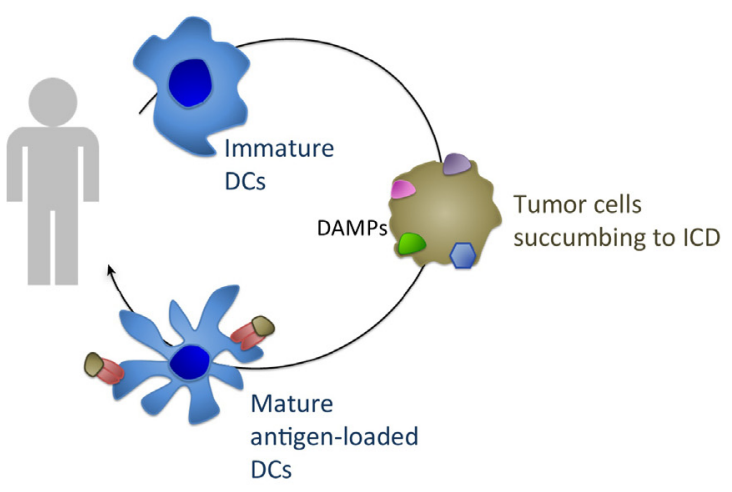

Trends in Cance

Figure 5. The antitumorigenic potential of single approaches may be synergically increased by combining ICD with other immunotherapies under clinical development resulting in a more effective reinstatement of a competent antitumor immunity. (A) Coadministration of anti-CD47 antibody would shift the balance toward 'eat-me' signal provided by exposure of CRT upon ICD. (B) ICD cannot completely subvert the immunosuppressed status of the TME, but can favor initial antitumor immunity responses. ICBs instead could favor the maintenance of an active pool of T cells by blocking the inhibitory signals provided by PD-L1/PD-1 and CTLA-4/B7. (C) The pool of tumor-infiltrating T cells used as source for adoptive transfer can be increased by neoadjuvant chemotherapy with ICD inducers. In addition, administration of ICD inducers after ACT could lead to reactivation of adoptively transferred T cells that persist in an inactive state in the circulation. (D) Incubation of autologous DCs with patient's cancer cells dying of ICD results in the generation of fully mature DCs without further need for maturation cocktails. ACT, adoptive T-cell transfer; CRT, calreticulin; CTLA, cytotoxic T-lymphocyte-associated protein; DC, dendritic cell; ER, endoplasmic reticulum; ICB, immune-checkpoint blocker; ICD, immunogenic cancer cell death; PD-1, programmed cell death-1; PD-L1, programmed death-ligand 1; TME, tumor microenvironment.

In addition, another hurdle to the application of ICD in the clinic is the usage of therapeutics close to the maximum-tolerated dose or in multiple cycles, which while being effective in killing cancer cells tend to kill also the immune cells causing lympho- or leukopenia and consequently depleting the immune mediators of ICD. In this perspective, a lower or metronomic dosage, as employed in the case of neoadjuvant treatments or cyclophosphamide, respectively, can be 


\section{Trends in Cancer}

more advantageous in preserving the full ICD potential of these drugs [71]. By contrast, it should be noted that a transient lymphodepletion may be beneficial due to a resetting of the immune system and elimination of protumorigenic immunosuppressive cells [72].

Alternatively, to bypass therapy-induced immunosuppression, ICD can be exploited through an ex vivo DC-based vaccination setting. This latter approach entails usage of autologous DCs loaded with autologous tumor cells that have undergone ICD, followed by DC maturation via 'maturation cocktails' and reinfusion of this DC vaccine in the patient. It has been demonstrated that such ICD-based DC vaccines exhibit high preclinical and clinical efficacy, even against immunotherapy-refractory tumor types like glioblastoma [73,74]. Moreover, the likelihood of patient to respond to such immunotherapies is predictable through the examination of the ICDsusceptibility potential of respective patient-derived tumor cells [73]. ICD could also be coupled to adoptive T-cell transfer (ACT), in particular, in the case of the treatment of solid tumors. Indeed, on the one hand ICD could be used in a neoadjuvant setting to enrich the tumor with tumor-infiltrating lymphocytes that serve as source for subsequent ACT. On the other hand, ICD inducers could be administered after ACT, to restimulate ACT cells that have been reported to stay in the circulation in inactive state after a first wave of efficacious antitumor activity (Figure 5).

In conclusion, while the prospect of implementing ICD in the clinical practice is facilitated by the known limited safety concerns related to clinically used ICD inducers, reliable biomarkers for monitoring ICD induction in patients still need to be identified and validated. In addition, combination with currently used or next-generation immunotherapies will probably be required to fully harness the therapeutic benefit of immunogenic cancer cell death induction.

\section{Concluding Remarks}

The immune system represents the most powerful natural defense against tumors, as it recognizes and eliminates residual transformed cells. However, tumor cells succeed in escaping immunosurveillance through multiple expedients resulting not only in immune evasion, but also in tumor-supporting inflammation. As described so far, ER stress plays a crucial role in supporting the survival and immune evasion of cancer cells with multiple cell-autonomous and nonautonomous functions. However, our knowledge of the UPR mediators of cancer cell lifedeath decisions and signal integration in the face of the evolving TME is still limited and urges future studies defining the complex role of UPR biology in tumors (see Outstanding Questions).

By contrast, it is clear that certain forms of therapy-induced ER stress can reinstate tumor immunogenicity through the induction ICD and the elicitation of ER stress-based danger signaling. Presumably, as discussed earlier, ER stress-mediated ICD as a monotherapy would not be able to endure T-cell-mediated antitumor immunity. Thus, a combinatorial therapeutic strategy, for example, combining ICD with immune checkpoint inhibitors, appears to be preferable and some recent preclinical studies indeed support this view. Careful consideration on the timing and design of the combinatorial therapy is furthermore needed to move rapidly toward the clinical setting. Finally, considering the emerging role of UPR pathways in governing key functions of various immune cells, such as antigen-presenting cells, the full appreciation of the immunological TME landscape regulated by ER stress pathways at the interface between cancer cells and immune cells is still largely in its infancy (see Outstanding Questions).

\section{Acknowledgments}

N.R. is funded by European Union's Horizon 2020 research and innovation programme under the Marie Sklodowska-Curie grant agreement no. 642295. A.D.G. is a recipient of the FWO postdoctoral fellowship 2013. This work is supported by grants from FWO (G060713N, G076617N), KU Leuven (C16/15/073) to P.A.

\section{Outstanding Questions}

What is the precise mechanism underlying the engagement of a prosurvival rather than a proapoptotic program upon ER stress?

Is PERK the only UPR component necessary for inducing ICD, or do IRE1 $\alpha$ and ATF6 also have a role?

Why concomitant ER stress and ROS are necessary for inducing ICD?

Are other cell death modalities able to generate an adaptive immune response, and is ER stress relevant in this context?

Which clinical biomarkers would be most suitable to evaluate ICD as well as have positive predictive value for therapy responses in cancer patients?

Which molecular determinants could reliably discriminate between ICD and non-ICD inducers?

What would be the optimal clinical protocol for the combinatorial administration of ICD inducers and antibodies aimed at immune checkpoint blockade? 


\section{Trends in Cancer}

\section{References}

1. Araki, K. and Nagata, K. (2011) Protein folding and quality control in the ER. Cold Spring Harb. Perspect. Biol. 3, a007526

2. Xu, C. et al. (2005) Endoplasmic reticulum stress: cell life and death decisions. J. Clin. Invest. 115, 2656-2664

3. Hetz, C. et al. (2013) Targeting the unfolded protein response in disease. Nat. Rev. Drug Discov. 12, 703-719

4. Ron, D. and Walter, P. (2007) Signal integration in the endoplasmic reticulum unfolded protein response. Nat. Rev. Mol. Cell Biol. 8, 519-529

5. Kimata, Y. and Kohno, K. (2011) Endoplasmic reticulum stresssensing mechanisms in yeast and mammalian cells. Curr. Opin. Cell Biol. 23, 135-142

6. Maurel, M. et al. (2015) Controlling the unfolded protein responsemediated life and death decisions in cancer. Semin. Cancer Biol. 33, 57-66

7. Han, J. et al. (2013) ER-stress-induced transcriptional regulation increases protein synthesis leading to cell death. Nat. Cell Biol. $15,481-490$

8. Savelieva, S. et al. (2015) Endoplasmic reticulum stress induces ligand-independent TNFR1-mediated necroptosis in L929 cells. Cell Death. Dis. 6, e1587

9. Dixon, S.J. et al. (2014) Pharmacological inhibition of cystineglutamate exchange induces endoplasmic reticulum stress and ferroptosis. Elife 2014, 1-25

10. Janssens, S. et al. (2014) Emerging functions of the unfolded protein response in immunity. Nat. Immunol. 15, 910-919

11. Bettigole, S.E. and Glimcher, L.H. (2014) Endoplasmic reticulum stress in immunity. Annu. Rev. Immunol. 33, 107-138

12. Urra, H. et al. (2016) Endoplasmic reticulum stress and the hallmarks of cancer. Trends Cancer 2, 252-262

13. Garg, A.D. et al. (2015) Targeting the hallmarks of cancer with therapy-induced endoplasmic reticulum (ER) stress. Mol. Cell. Oncol. 2, e975089

14. Kato, H. and Nishitoh, H. (2015) Stress responses from the endoplasmic reticulum in cancer. Front. Oncol. 5, 1-15

15. Hazari, Y.M. et al. (2016) Emerging tale of UPR and cancer: an essentiality for malignancy. Tumor Biol. 37, 14381-14390

16. Giampietri, C. et al. (2015) Cancer microenvironment and endoplasmic reticulum stress response. Mediators Inflamm. 2015, 417281

17. Rodvold, J.J. et al. (2016) Immune modulation by ER stress and inflammation in the tumor microenvironment. Cancer Lett. 380, $227-236$

18. Binet, F. and Sapieha, P. (2015) ER stress and angiogenesis. Cell Metab. 22, 560-575

19. Hanahan, D. and Weinberg, R.A. (2011) Hallmarks of cancer: the next generation. Cell 144, 646-674

20. Blankenstein, T. et al. (2012) The determinants of tumour immunogenicity. Nat. Rev. Cancer 12, 307-313

21. Wynn, T.A. (2015) Type 2 cytokines: mechanisms and therapeutic strategies. Nat. Rev. Immunol. 15, 271-282

22. Deng, J. et al. (2004) Translational repression mediates activation of nuclear factor kappa B by phosphorylated translation initiation factor 2. Mol. Cell Biol. 24, 10161-10168

23. Mantovani, A. et al. (2008) Cancer-related inflammation. Nature $454,436-444$

24. Hu, P. et al. (2006) Autocrine tumor necrosis factor alpha links endoplasmic reticulum stress to the membrane death receptor pathway through IRE1alpha-mediated NF-B activation and down-regulation of TRAF2 expression. Society 26, 3071-3084

25. Urano, F. et al. (2000) Coupling of stress in the ER to activation of JNK protein kinases by transmembrane protein kinase IRE1. Science 287, 664-666

26. Yamazaki, H. et al. (2009) Activation of the Akt-NF-kappaB pathway by subtilase cytotoxin through the ATF6 branch of the unfolded protein response. J. Immunol. 183, 1480-1487

27. Menu, P. et al. (2012) ER stress activates the NLRP3 inflammasome via an UPR-independent pathway. Cell Death. Dis. 3, e261

28. Lebeaupin, C. et al. (2015) ER stress induces NLRP3 inflammasome activation and hepatocyte death. Cell Death. Dis. 6, e1879
29. Grivennikov, S.I. et al. (2010) Immunity, inflammation, and cancer. Cell 140, 883-899

30. Grivennikov, S. and Karin, M. (2008) Autocrine IL-6 signaling: a key event in tumorigenesis? Cancer Cell 13, 7-9

31. Qian, X. et al. (2013) Increased Th17 cells in the tumor microenvironment is mediated by IL-23 via tumor-secreted prostaglandin E2. J. Immunol. 190, 5894-5902

32. Hung, J.H. et al. (2004) Endoplasmic reticulum stress stimulates the expression of cyclooxygenase-2 through activation of NFkappaB and pp38 mitogen-activated protein kinase. J. Biol. Chem. 279, 46384-46392

33. Mahadevan, N.R. and Zanetti, M. (2011) Tumor stress inside out: cell-extrinsic effects of the unfolded protein response in tumo cells modulate the immunological landscape of the tumor microenvironment. J. Immunol. 187, 4403-4409

34. Mahadevan, N.R. et al. (2011) Transmission of endoplasmic reticulum stress and pro-inflammation from tumor cells to myeloid cells. Proc. Natt. Acad. Sci. U. S. A. 108, 6561-6566

35. Rausch, M.P. and Sertil, A.R. (2015) A stressful microenvironment: opposing effects of the endoplasmic reticulum stress response in the suppression and enhancement of adaptive tumor immunity. Int. Rev. Immunol. 34, 104-122

36. Schäfer, M. and Werner, S. (2008) Cancer as an overhealing wound: an old hypothesis revisited. Nat. Rev. Mol. Cell Biol. 9 , 628-638

37. Poon, I.K. et al. (2014) Apoptotic cell clearance: basic biology and therapeutic potential. Nat. Rev. Immunol. 14, 166-180

38. Green, D.R. et al. (2009) Immunogenic and tolerogenic cell death. Nat. Rev. Immunol. 9, 353-363

39. Tesniere, A. et al. (2008) Molecular characteristics of immunogenic cancer cell death. Cell Death. Differ. 15, 3-12

40. Krysko, D.V. et al. (2012) Immunogenic cell death and DAMPs in cancer therapy. Nat. Rev. Cancer 12, 860-875

41. Obeid, M. et al. (2007) Calreticulin exposure dictates the immunogenicity of cancer cell death. Nat. Med. 13, 54-61

42. Garg, A.D. et al. (2014) Danger signalling during cancer cell death: origins, plasticity and regulation. Cell Death. Differ. 21, 26-38

43. Ghiringhelli, F. et al. (2009) Activation of the NLRP3 inflammasome in dendritic cells induces IL-1beta-dependent adaptive immunity against tumors. Nat. Med. 15, 1170-1178

44. Apetoh, L. et al. (2007) Toll-like receptor 4-dependent contribution of the immune system to anticancer chemotherapy and radiotherapy. Nat. Med. 13, 1050-1059

45. Garg, A.D. et al. (2017) Pathogen response-like recruitment and activation of neutrophils by sterile immunogenic dying cells drives neutrophil-mediated residual cell killing. Cell Death. Differ. 24, 832-843

46. Vacchelli, E. et al. (2015) Chemotherapy-induced antitumor immunity requires formyl peptide receptor 1 . Science 350 972-978

47. Sistigu, A. et al. (2014) Cancer cell-autonomous contribution of type I interferon signaling to the efficacy of chemotherapy. Nat Med. 20, 1301-1309

48. Garg, A.D. et al. (2015) Molecular and translational classification of DAMPs in immunogenic cell death. Front. Immunol. 6, 588

49. Bezu, L. et al. (2015) Combinatorial strategies for the induction of immunogenic cell death. Front. Immunol. 6, 187

50. Aaes, T.L. et al. (2016) Vaccination with necroptotic cancer cells induces efficient anti-tumor immunity. Cell Rep. 15, 1-14

51. Yang, H. et al. (2016) Contribution of RIP3 and MLKL to immunogenic cell death signaling in cancer chemotherapy. Oncoimmunology 5, e1149673

52. Garg, A.D. et al. (2012) A novel pathway combining calreticulin exposure and ATP secretion in immunogenic cancer cell death. EMBO J. 31, 1062-1079

53. Koks, C.A. et al. (2015) Newcastle disease virotherapy induces long-term survival and tumor-specific immune memory in orthotopic glioma through the induction of immunogenic cell death. Int. J. Cancer 136, E313-E325 


\section{Trends in Cancer}

54. Miyamoto, S. et al. (2012) Coxsackievirus B3 is an oncolytic virus with immunostimulatory properties that is active against lung adenocarcinoma. Cancer Res. 72, 2609-2621

55. Wong, D.Y. et al. (2015) Induction of immunogenic cell death by chemotherapeutic platinum complexes. Angew. Chem. Int. Ed. Engl. 54, 6483-6487

56. Moserova, I. et al. (2017) Caspase-2 and oxidative stress underlie the immunogenic potential of high hydrostatic pressure-induced cancer cell death. Oncoimmunology 6, e1258505

57. Van Vliet, A.R. et al. (2017) The ER stress sensor PERK coordinates ER-plasma membrane contact site formation through interaction with Filamin-A and F-actin remodeling. Mol. Cell 65, $1-15$

58. Panaretakis, T. et al. (2009) Mechanisms of pre-apoptotic calreticulin exposure in immunogenic cell death. EMBO J. 28, 578590

59. Porat-Shliom, N. et al. (2013) Multiple roles for the actin cytoskeleton during regulated exocytosis. Cell. Mol. Life Sci. 70, 2099 2121

60. Pol, J. et al. (2015) Trial watch: immunogenic cell death inducers for anticancer chemotherapy. Oncoimmunology 4 e1008866

61. Garg, A.D. et al. (2016) Immunological metagene signatures derived from immunogenic cancer cell death associate with improved survival of patients with lung, breast or ovarian malignancies: a large-scale meta-analysis. Oncoimmunology 5, e1069938

62. Ladoire, S. et al. (2016) Biomarkers of immunogenic stress in metastases from melanoma patients: correlations with the immune infiltrate. Oncoimmunology 5, e1160193

63. Gebremeskel, S. and Johnston, B. (2015) Concepts and mechanisms underlying chemotherapy induced immunogenic cell death: impact on clinical studies and considerations for combined therapies. Oncotarget 6, 41600-41619

64. Garg, A.D. et al. (2015) Resistance to anticancer vaccination effect is controlled by a cancer cell-autonomous phenotype that disrupts immunogenic phagocytic removal. Oncotarget 6, 26841-26860

65. Fucikova, J. et al. (2015) Prognostic and predictive value of DAMPs and DAMP-associated processes in cancer. Front. Immunol. 6, 402

66. Liu, X. et al. (2015) CD47 blockade triggers T cell-mediated destruction of immunogenic tumors. Nat. Med. 21, 1209-1215

67. Van Allen, E.M. et al. (2015) Genomic correlates of response to CTLA-4 blockade in metastatic melanoma. Science 350, $207-$ 211

68. Rizvi, N.A. et al. (2015) Mutational landscape determines sensitivity to PD-1 blockade in non-small cell lung cancer. Science 348 , $124-128$

69. Shin, D.S. and Ribas, A. (2015) The evolution of checkpoint blockade as a cancer therapy: what's here, what's next? Curr. Opin. Immunol. 33, 23-35

70. Pfirschke, C. et al. (2016) Immunogenic chemotherapy sensitizes tumors to checkpoint blockade therapy. Immunity 44, 343-354
71. Galluzzi, L. et al. (2015) Immunological effects of conventiona chemotherapy and targeted anticancer agents. Cancer Cell 28, 690-714

72. Emens, L.A. (2010) Chemoimmunotherapy. Cancer J. 16, 295303

73. Zappasodi, R. et al. (2010) Improved clinical outcome in indolent B-cell lymphoma patients vaccinated with autologous tumor cells experiencing immunogenic death. Cancer Res. 70, 9062-9072

74. Garg, A.D. et al. (2016) Dendritic cell vaccines based on immunogenic cell death elicit danger signals and T cell-driven rejection of high-grade glioma. Sci. Transl. Med. 8, 27-328

75. Ladoire, S. et al. (2015) Combined evaluation of LC3B puncta and HMGB1 expression predicts residual risk of relapse after adjuvant chemotherapy in breast cancer. Autophagy 11, 1878-1890

76. Suzuki, Y. et al. (2012) Immunogenic tumor cell death induced by chemoradiotherapy in patients with esophageal squamous cell carcinoma. Cancer Res. 72, 3967-3976

77. Liikanen, I. et al. (2015) Serum HMGB1 is a predictive and prognostic biomarker for oncolytic immunotherapy. Oncoimmunology 4, e989771

78. Wemeau, M. et al. (2010) Calreticulin exposure on malignant blasts predicts a cellular anticancer immune response in patients with acute myeloid leukemia. Cell Death Dis. 1, e104

79. Peng, R.Q. et al. (2010) Expression of calreticulin is associated with infiltration of T-cells in stage III B colon cancer. World J. Gastroenterol. 16, 2428-2434

80. Senovilla, L. et al. (2012) An immunosurveillance mechanism controls cancer cell ploidy. Science 337, 1678-1684

81. Schardt, J.A. et al. (2009) Activation of the unfolded protein response is associated with favorable prognosis in acute myeloid leukemia. Clin. Cancer Res. 15, 3834-3841

82. Mozos, A. et al. (2011) The expression of the endoplasmic reticulum stress sensor BiP/GRP78 predicts response to chemotherapy and determines the efficacy of proteasome inhibitors in diffuse large B-cell lymphoma. Am. J. Pathol. 179, 2601-2610

83. Hetz, C. (2012) The unfolded protein response: controlling cell fate decisions under ER stress and beyond. Nat. Rev. Mol. Cell Biol. 13, 89-102

84. Meares, G.P. et al. (2014) PERK-dependent activation of JAK and STAT3 contributes to endoplasmic reticulum stress-induced inflammation. Mol. Cell. Biol. 34, 3911-3925

85. Philippe, L. et al. (2013) The miR-17 92 cluster: a key player in the control of inflammation during rheumatoid arthritis. Front Immunol. 4, 1-5

86. Lerner, A.G. et al. (2012) IRE1 $\alpha$ induces thioredoxin-interacting protein to activate the NLRP3 inflammasome and promote programmed cell death under irremediable ER stress. Cell Metab. $16,250-264$

87. Michaud, M. et al. (2011) Autophagy-dependent anticance immune responses induced by chemotherapeutic agents in mice. Science 334, 1573-1577

88. Kepp, O. et al. (2014) Consensus guidelines for the detection of immunogenic cell death Oncoimmunology 3, e955691 